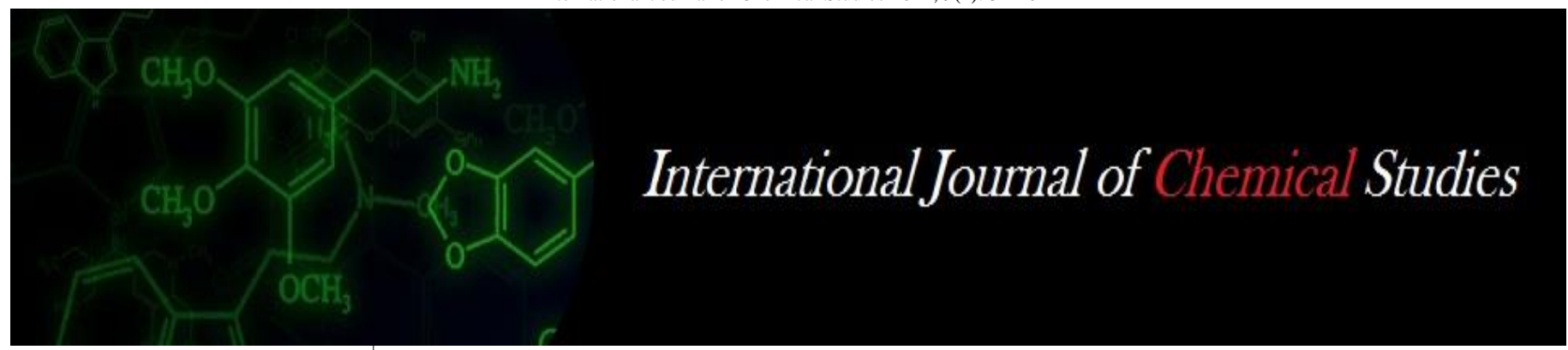

P-ISSN: 2349-8528

E-ISSN: 2321-4902

www.chemijournal.com

IJCS 2021; 9(1): 34-40

(C) 2021 IJCS

Received: 12-11-2020

Accepted: 19-12-2020

Ibrahim Mahmoud Ahmed Ibrahim

Department of Special Food and Nutrition, Food Technology Research Institute, Agriculture Research Center, Giza, Egypt

Corresponding Author: Ibrahim Mahmoud Ahmed Ibrahim

Department of Special Food and Nutrition, Food Technology Research Institute, Agriculture Research Center, Giza, Egypt

\section{Assessment of the physicochemical and sensorial features of pasteurized cantaloupe juice during storage}

\author{
Ibrahim Mahmoud Ahmed Ibrahim
}

DOI: https://doi.org/10.22271/chemi.2021.v9.i1a.11595

\begin{abstract}
The study evaluated the effect of pasteurization temperatures and storage methods on physicochemical properties and sensory attributes of cantaloupe juice. Cantaloupe juice was pasteurized at $\left(85^{\circ} \mathrm{C} / 90 \mathrm{sec}\right.$ and $95{ }^{\circ} \mathrm{C} / 45 \mathrm{sec}$ ) and the juice was stored at $\left(4^{\circ} \mathrm{C}\right.$ and room temperature) for 28 days. Pasteurization at $95{ }^{\circ} \mathrm{C}$ for 45 seconds has a good effect on nutritional values compared with pasteurization at $85{ }^{\circ} \mathrm{C}$ for 90 seconds. The refrigerator storage at $4{ }^{\circ} \mathrm{C}$ for 28 days allowed maintaining a higher level of quality parameters compared to ambient storage. The results of HPLC analysis confirmed that flavonoids are primarily responsible for the antioxidant activity in the cantaloupe juice, which accounts for $43.71 \%$ of total phenolic compounds in the cantaloupe juice. Pasteurization at $85{ }^{\circ} \mathrm{C} / 90 \mathrm{sec}$ harmed sensory attributes while pasteurization at $95{ }^{\circ} \mathrm{C} / 45 \mathrm{sec}$ maintained all sensory attributes. Also, the increase in the storage period leads to a noticeable decrease in the sensory attributes.
\end{abstract}

Keywords: Cantaloupe, pasteurized, refrigerated, sensory attributes, physicochemical properties, storage

\section{Introduction}

The bioactive compounds such as vitamin $\mathrm{C}$, carotenoids, total phenols, and flavonoids existing in fruit and vegetables restrict many risks of diseases, for biological impacts on public health (Garavand et al., 2019; Harwansh et al., 2019) ${ }^{[10,12]}$. Also, it known by the ability of food protection purposes and many influences, like antimicrobial, antihypertensive, and antioxidant properties (Banožić et al., 2020; Oliveira et al., 2020) ${ }^{[3,15] .}$

Bioactive compounds existing in rich foods and drinks lessen the danger of generating chronic diseases like cardiovascular disease, atherosclerosis, cancer, diabetes, cataracts, cognitive control diseases, and neurological disorders (Biazotto et al., 2019; Murador et al. 2019; Santamarina et al., 2019; Mesquita et al., 2020) ${ }^{[5,22,26,18] .}$

Cantaloupe (Cucumis melo L.) has a distinct flavor that makes it a popular crop to consumers. It is moderate quantities of bioactive substances, including phenolic compounds, vitamin $\mathrm{C}$, pro-vitamin A ( $\beta$-carotene), and folic acid, but is considered to have significant human health implications (Laur and Tian, 2011) ${ }^{[16]}$. The higher intake of carotenoids is associated with protection from chronic diseases like cardiovascular disease and carcinoma (Wang et al., 2011) ${ }^{[32]}$. Phenolic compounds include a significant benefit in keeping healthy (Paul et al., 2019) ${ }^{[23]}$. Cantaloupe honeydew contained 59.3-109.6 mg/100 of those component groups (INRA, 2009) ${ }^{[13]}$. Benzoic, vanilla and trans-cinnamic acids are the main phenols present in cantaloupes in large amounts, with small concentrations of p-coumaric acid, gallic acid, phydroxybenzoic acid, syringic acid, epicatechin, ferulic acid protocatechuic acid, quercetin, chlorogenic acid, and routine in cantaloupe juice (Kolayli et al., 2010) ${ }^{[14]}$.

Thermal technologies are used for juices to ensure enzyme inactivation, particularly at a pH of less than 4.5. This study investigates the influence of different pasteurization temperatures and storage periods at diverse temperatures on the initial quality attributes of cantaloupe juice. 
Materials and Methods

Materials and chemicals

Cantaloupe (Cucumis melo L. var. Bander) was collected from Ismailia, Egypt. The employed chemicals and reagents in this study were analytical grade brought from Sigma firm (St. Louis, USA)

\section{Methods}

\section{Processing of cantaloupe}

The fruits of cantaloupe were washed with tap water, drained until dried, peeled, and cut into small pieces, the juice got by Moulinex extractor (T574, France). The juice $\mathrm{pH}$ adjusted to pH 4 after 6.36 for fresh, the juice is divided into two flasks $(500 \mathrm{ml})$, the primary flask heated to $90^{\circ} \mathrm{C}$ by boiling water $\left(100^{\circ} \mathrm{C}\right)$ within the first water bath, and then put in another water bath adjusted at $90^{\circ} \mathrm{C}$ to stay the sample for 45 seconds. Similarly, the cantaloupe juice within the second flask pasteurized at $85^{\circ} \mathrm{C}$ for 90 seconds,

Table 1: The symbols are used for different treatments

\begin{tabular}{|c|c|}
\hline Treatments & symbols \\
\hline Fresh & $\mathrm{F}$ \\
\hline Pasteurized $85^{\circ} \mathrm{C} / 90 \mathrm{sec}+$ ambient storage & $\mathrm{F} 1$ \\
\hline Pasteurized $85^{\circ} \mathrm{C} / 90 \mathrm{sec}+$ Storage at $4{ }^{\circ} \mathrm{C}$ & $\mathrm{F} 2$ \\
\hline Pasteurized $90^{\circ} \mathrm{C} / 45 \mathrm{sec}+$ ambient storage & $\mathrm{F} 3$ \\
\hline Pasteurized $90^{\circ} \mathrm{C} / 45 \mathrm{sec}+$ Storage at $4{ }^{\circ} \mathrm{C}$ & $\mathrm{F} 4$ \\
\hline
\end{tabular}

$90 \mathrm{ml}$ of pasteurized juice placed in $100 \mathrm{ml}$ of sealed sterile glass containers and stored under refrigerated conditions $(4 \pm$ $\left.2{ }^{\circ} \mathrm{C}\right)$ and at room temperature $\left(25 \pm 5{ }^{\circ} \mathrm{C}\right)$ for 28 days. The analyses were achieved for fresh, after processing, and weekly after that throughout 28 days. The symbols used in various treatments are seen in Table 1.

\section{Physicochemical analysis}

Measurement of total soluble solids (TSS), pH, titrable acidity (TA), and vitamin C (VC)

TSS were estimated as Brix degrees using an Abbe refractometer $\left(\mathrm{C} 10\right.$, Vee Gee, USA) at $20^{\circ} \mathrm{C}$. The $\mathrm{pH}$ was monitored by $\mathrm{pH}$ meter Jenway 510 (Bibby Scientific Ltd. Stone, Staff, UK). TA and VC were calculated identical to (AOAC, 2000) $)^{[2]}$.

Measurement of the browning index, total carotenoids (TC), and color parameters

Browning index was assessed by Meydov et al. (1977) [20] method as absorbance at $420 \mathrm{~nm}$. TC determination was achieved by Barros et al. (2011) ${ }^{[4]}$ methods as $\beta$-carotene. Color parameters (CIE L*, $\mathrm{a}^{*}$, and $\mathrm{b}^{*}$ ) were performed by Konika Minolta reader (CR-10, Inc., Osaka, Japan). Hue angle and chroma were calculated from $\mathrm{a}^{*}$ and $\mathrm{b}^{*}$ values.

\section{Determination of antioxidant activity (AA) and total phenolics (TP)}

The DPPH method was employed for AA determination according to Ravichandran et al. (2013) ${ }^{[25]}$. TP was calculated as stated by Ferrante et al. (2004) and Spadafora et al. $(2016)^{[8,28]}$.

\section{Fractionation of phenolic compounds using HPLC}

Phenolic components fractionated by HPLC as described by
Goupy et al. (1999) and Mattila et al. (2000) ${ }^{[11,17]} .5$ grams of cantaloupe juice were centrifuged for $10 \mathrm{~min}$ with methanol at $1000 \mathrm{rpm}$. A 0.2-micrometer membrane filter is employed to obtain 1-3 ml extract to injection in HPLC Hewllet Packard (Series 1050) with an automatic sample injector, solvent degassed, ultraviolet detector adjusted at $280 \mathrm{~nm}$, and a quarter pump HP (series1050). Phenols identified by C18 (BDS $5 \mu \mathrm{m}$, Labio, Czech Republic) column (250 x $4 \mathrm{~mm}$, i.d.). Acetonitrile and methanol were used as a gradient separation at flow rate $1 \mathrm{ml} / \mathrm{min}$, and the column was kept at $35^{\circ} \mathrm{C}$. Thirteen standard phenolic compounds from Sigma Co., (St. Louis, USA) were injected into HPLC. Retention time and peak area were used to calculate phenolics concentration from the 63 data analysis of Hewllet Packed software.

\section{Sensory evaluation}

The method of Min et al. (2003) [19] was used for sensory attributes determination. Ten panelists of Food Technology Institute members, Ismailia, Egypt, conducted a sensory assessment. The panelists assign a suitable score for characteristic color, taste, odor, appearance, mouthfeel, and overall acceptability from 9 points.

\section{Statistical analysis}

All values were analyzed in triplicate and represented as means \pm SD (standard deviation). Statistical analyses of data were performed with the COSTAT software (version 6311) at $\mathrm{P} \leq 0.05 .3$.

\section{Results}

The effects of various pasteurization temperatures and storage on TSS, pH, acidity, and absorption at $420 \mathrm{nn}$, ascorbic acid, total phenols, carotenoids, and AA of cantaloupe juice are revealed in Table 2.

\section{TSS}

No significant changes were initiated in TSS among all treatments used and the fresh sample at zero time. TSS increased significantly during storage for 4 weeks in the whole treatments. F4 did not record any changes after 4 weeks compared with the fresh sample and their value at zero time. The importance of chilled storing and pasteurization at $90{ }^{\circ} \mathrm{C}$ on maintaining TSS from change has appeared from the results.

\section{pH and acidity}

There were no major variations in $\mathrm{pH}$ and acidity in treated samples at zero time. By storage ending, the chilled sample (F4 and F2) held the minimal acidity of cantaloupe juice $(0.44 \pm 0.03, \quad 0.47 \pm 0.04)$, with no noticeable difference between them and the fresh sample $(0.38 \pm 0.06)$.

\section{Browning (absorption at 420)}

As regards absorption at 420 cantaloupe juice, increasing pasteurization temperatures to $90^{\circ} \mathrm{C}$, as in $(\mathrm{F} 3, \mathrm{~F} 4)$ increasing absorption at 420 at zero, while pasteurization at $\left(85^{\circ} \mathrm{C}\right)$ showed a mild rise in cantaloupe juice browning. The increase in browning continued during storage, with lower browning of the refrigerated samples (F4, F2), especially F4 with a value $(0.158 \pm 0.15)$. 


\section{Vitamin C}

Table 2: Impact of various pasteurized temperatures and storage on TSS, pH, acidity, absorption, vitamin C, total phenols, carotenoids, and DPPH of cantaloupe juice

\begin{tabular}{|c|c|c|c|c|c|c|c|c|c|}
\hline $\mathrm{Sa}$ & \begin{tabular}{|c|}
$\begin{array}{c}\text { Storage } \\
\text { weeks }\end{array}$ \\
\end{tabular} & TSS\% & pH & y\% & ssorbation 420 & $\begin{array}{c}\text { vitamin } C \\
\mathrm{mg} / 100 \mathrm{~g}\end{array}$ & $\begin{array}{c}\text { T. phenols } \\
\text { mg/100g }\end{array}$ & $\begin{array}{c}\text { Carotenoids } \\
\mu \mathrm{g} / \mathbf{1 0 0 \mathrm { g }}\end{array}$ & DРPH\% \\
\hline $\mathrm{F}$ & Fresh & $10.41 \pm 0.22^{\mathrm{f}}$ & $6.36 \pm 0.05^{\mathrm{a}}$ & $0.38 \pm 0.06^{\mathrm{bcd}}$ & $0.089 \pm 0.012^{\mathrm{m}}$ & $56.54 \pm 0.05^{\mathrm{a}}$ & $127.01 \pm 0.38^{\mathrm{a}}$ & $817.42 \pm 0.32^{\mathrm{a}}$ & $34.9 \pm 0.35^{\text {ghi }}$ \\
\hline \multirow{5}{*}{$\mathrm{F} 1$} & 0 time & $10.42 \pm 0.13^{\mathrm{f}}$ & $4.00 \pm 0.06^{\mathrm{b}}$ & $0.37 \pm 0.05^{\text {bcd }}$ & $0.098 \pm 0.005^{\mathrm{im}}$ & $50.18 \pm 0.16^{\mathrm{c}}$ & $113.24 \pm 0.39^{\mathrm{f}}$ & $802.67 \pm 0.37^{c}$ & $37.41 \pm 0.24^{\mathrm{e}}$ \\
\hline & 1week & $10.55 \pm 0.21^{\text {cdef }}$ & $3.97 \pm 0.05^{\mathrm{b}}$ & $0.41 \pm 0.03^{a b c d}$ & $0.119 \pm 0.003^{\mathrm{jk}}$ & $47.16 \pm 0.13^{\mathrm{g}}$ & $103.84 \pm 0.08^{\mathrm{i}}$ & $769.12 \pm 0.18^{\mathrm{g}}$ & $35.91 \pm 0.48^{f}$ \\
\hline & 2 week & $10.73 \pm 0.14^{\text {abcdef }}$ & $3.92 \pm 0.04^{\mathrm{b}}$ & $0.44 \pm 0$ & $7^{\text {efg }}$ & $44.01 \pm 0.08^{\mathrm{k}}$ & $92.65 \pm 0.05^{\mathrm{m}}$ & $0.62^{1}$ & $27^{\text {ghi }}$ \\
\hline & 3Week & \begin{tabular}{|l|}
$10.84 \pm 0.09^{\mathrm{abc}}$ \\
\end{tabular} & $3.89 \pm 0.12^{\mathrm{a}}$ & $0.48 \pm 0.03^{\mathrm{ab}}$ & $3 \pm 0.009^{\mathrm{bc}}$ & 39.82 & 82.79 & $.35^{\mathrm{p}}$ & $35^{\mathrm{jk}}$ \\
\hline & 4week & 10.9 & $3.89 \pm 0$ & & & 36.0 & $.24^{\mathrm{q}}$ & $3.32^{\mathrm{r}}$ & $.43^{1}$ \\
\hline \multirow{5}{*}{$\mathrm{F} 2$} & 0 time & $10.43 \pm 0.16^{\mathrm{ef}}$ & $4.00 \pm 0.07^{\mathrm{b}}$ & $0.37 \pm 0.04^{\text {bcd }}$ & $0.096 \pm 0.011^{1 \mathrm{~m}}$ & $51.23 \pm 0.08^{\mathrm{b}}$ & $114.85 \pm 0.05^{\mathrm{d}}$ & $801.37 \pm 0.51^{\mathrm{c}}$ & $36.84 \pm 0.31^{\mathrm{e}}$ \\
\hline & 1week & $10.48 \pm 0.15^{\text {def }}$ & $4.00 \pm 0.04^{\mathrm{b}}$ & $0.39 \pm 0.05^{\text {bcd }}$ & $0.123 \pm 0.012^{\mathrm{ijk}}$ & $49.60 \pm 0.12^{\mathrm{d}}$ & $107.75 \pm 0.04^{\mathrm{g}}$ & $776.42 \pm 0.29^{\mathrm{e}}$ & $35.06 \pm 0.05^{\mathrm{gh}}$ \\
\hline & 2 week & $10.59 \pm 0.18^{\text {bcdef }}$ & $3.98 \pm 0.05^{\mathrm{b}}$ & $0.41 \pm 0.09^{\text {abcd }}$ & $0.155 \pm 0.008^{\mathrm{efg}}$ & $44.67 \pm 0.07^{j}$ & 102.6 & $0.47^{\mathrm{i}}$ & $0.13^{\mathrm{hij}}$ \\
\hline & 3Week & $10.76 \pm 0.32^{\text {abcd }}$ & $3.96 \pm 0.08^{\mathrm{b}}$ & $0.44 \pm$ & $5^{\mathrm{de}}$ & 42.1 & 92.72 & $62^{\mathrm{m}}$ & $.34^{\mathrm{j}}$ \\
\hline & 4week & $10.76 \pm 0.12^{\text {abcd }}$ & $3.95 \pm 0.09^{\mathrm{b}}$ & $0.47 \pm$ & $7^{\mathrm{cd}}$ & $39.60 \pm 0.03^{q}$ & $84.40 \pm 0.28^{\circ}$ & $697.64 \pm 0.77^{\circ}$ & $32.78 \pm 0.67^{\mathrm{k}}$ \\
\hline \multirow{5}{*}{ F3 } & 0 time & $10.45 \pm 0.14^{\mathrm{def}}$ & $4.00 \pm 0.06^{\mathrm{b}}$ & $0.35 \pm 0.06^{\mathrm{d}}$ & $0.119 \pm 0.006^{\mathrm{jk}}$ & $47.08 \pm 0.11^{\mathrm{g}}$ & $122.16 \pm 0.32^{\mathrm{c}}$ & $806.75 \pm 0.84^{b}$ & $39.75 \pm 0.40^{\mathrm{ab}}$ \\
\hline & 1week & $10.53 \pm 0.09^{\text {cdef }}$ & $3.99 \pm 0.05^{\mathrm{b}}$ & $0.38 \pm 0.07^{b c d}$ & $0.138 \pm 0.008^{\mathrm{ghi}}$ & $49.50 \pm 0.10^{\mathrm{d}}$ & $113.43 \pm 0.36^{\mathrm{f}}$ & $771.66 \pm 2.06^{\mathrm{f}}$ & $38.92 \pm 0.29^{\mathrm{bc}}$ \\
\hline & 2 week & $10.69 \pm 0.17^{\text {abcdef }}$ & $3.97 \pm 0.04^{\mathrm{b}}$ & $0.43 \pm 0.03^{\text {abcd }}$ & $0.161 \pm 0.013^{\text {ef }}$ & $46.81 \pm 0.14^{\mathrm{h}}$ & $105.94 \pm 0.44^{\mathrm{h}}$ & $735.84 \pm 0.35^{j}$ & $37.42 \pm 0.23^{\mathrm{e}}$ \\
\hline & 3Week & $10.75 \pm 0.25^{\text {abcde }}$ & $3.96 \pm 0.10^{\mathrm{b}}$ & $0.48 \pm 0.09^{\mathrm{ab}}$ & $0.184 \pm 0.014^{\mathrm{cd}}$ & 40.0 & 90.3 & $.20^{\mathrm{n}}$ & $95^{\mathrm{fg}}$ \\
\hline & 4week & $10.88 \pm 0.15^{\mathrm{ab}}$ & $3.93 \pm 0.06^{\mathrm{b}}$ & $0.51 \pm$ & $0.204 \pm 0.004^{\mathrm{b}}$ & $37.18 \pm 0.22^{\mathrm{r}}$ & 87.27 & $677.41 \pm 0.82^{\mathrm{q}}$ & $0.71^{\mathrm{ij}}$ \\
\hline \multirow{5}{*}{ F4 } & 0 time & \begin{tabular}{|l|}
$10.44 \pm 0.18^{\mathrm{def}}$ \\
\end{tabular} & $4.00 \pm 0.05^{\mathrm{b}}$ & $0.36 \pm 0.08^{\mathrm{cd}}$ & $0.111 \pm .005^{\mathrm{kl}}$ & $49.13 \pm 0.07^{\mathrm{e}}$ & $124.62 \pm 0.22^{\mathrm{b}}$ & $805.57 \pm 0.34^{b}$ & $40.14 \pm 0.55^{\mathrm{a}}$ \\
\hline & 1week & $10.46 \pm 0.15^{\mathrm{def}}$ & $3.99 \pm 0.12^{\mathrm{b}}$ & $0.37 \pm 0.03^{\text {bcd }}$ & $0.124 \pm 0.020^{\mathrm{ijk}}$ & $47.75 \pm 0.04^{\mathrm{f}}$ & $114.24 \pm 0.42^{\mathrm{e}}$ & $787.74 \pm 0.17^{\mathrm{d}}$ & $39.15 \pm 0.64^{\mathrm{bc}}$ \\
\hline & 2 week & $10.49 \pm 0.12^{\text {def }}$ & $3.99 \pm 0.14^{\mathrm{b}}$ & $0.39 \pm 0.07^{b c d}$ & $0.132 \pm 0.007^{\text {hij }}$ & $45.15 \pm 0.14^{\mathrm{i}}$ & $107.81 \pm 0.09^{\mathrm{g}}$ & $765.65 \pm 0.33^{h}$ & $38.33 \pm 0.47^{\mathrm{cd}}$ \\
\hline & 3 Week & $10.53 \pm 0.08^{\text {cdef }}$ & $3.97 \pm 0.11^{\mathrm{b}}$ & $0.42 \pm 0.01^{\mathrm{abcd}}$ & $0.149 \pm 0.011^{\mathrm{fgh}}$ & $43.36 \pm 0.15^{1}$ & $100.43 \pm 0.08^{\mathrm{k}}$ & $749.71 \pm 0.15^{\mathrm{i}}$ & $37.54 \pm 0.47^{\mathrm{de}}$ \\
\hline & 4week & $10.56 \pm 0.10^{\text {cdef }}$ & $3.97 \pm 0.12^{\mathrm{b}}$ & $0.44 \pm 0.03^{\mathrm{abcd}}$ & $0.158 \pm 0.015^{\mathrm{ef}}$ & $41.54 \pm 0.06^{\mathrm{n}}$ & $94.65 \pm 0.14^{1}$ & $732.11 \pm 0.17^{\mathrm{k}}$ & $36.92 \pm 0.73^{\mathrm{e}}$ \\
\hline
\end{tabular}

The values are the mean \pm margin of confidence intervals at $95 \%$.

Data in the same column with different letters differ significantly $(p<0.05)$.

Vitamin $\mathrm{C}$ was decreased immediately after thermal treatment relative to the fresh sample. Over the storage cycle the destruction of vitamin $\mathrm{C}$ continues, but cold samples (F4, F2) retain significant quantities of vitamin $(41.54 \pm 0.06$; $39.60 \pm$ $0.03 \mathrm{mg} / 100 \mathrm{~g})(\mathrm{F} 1, \mathrm{~F} 3)$. The use of refrigerated storage with a higher degree of pasteurization $\left(90^{\circ} \mathrm{C}\right)$ resulted in a higher content of vitamin $\mathrm{C}$ after storage as $\mathrm{F} 4 \mathrm{did}$.

\section{Carotenoids}

The temperature used for pasteurization cantaloupe juice decreased carotenoid content compared to fresh samples. Pasteurized cantaloupe juice at $90^{\circ} \mathrm{C}$ for 45 seconds has a beneficial effect on carotenoids maintained at storage ending. Previous findings have declared the importance of pasteurization time on the maintenance of carotenoids. Carotenoids were decreased after 4 weeks of storage. The refrigerated samples, specifically F4, gave the lowest decrement by storage ending with a value of $732.11 \pm 0.17$, followed by $\mathrm{F} 2$ at $697.4 \pm 0.77$.

\section{Antioxidant activity\% (AA)}

The antioxidant function in fruit has returned to vitamin C, carotenoids, and polyphenols. The findings revealed that pasteurization in particular at $90^{\circ} \mathrm{C}$ increased the AA in cantaloupe juice after processing compared to the fresh sample. The destruction of cell membranes and walls by thermal processing-increased AA due to releases carotenoids from the insoluble components of the cantaloupe, which increases the bioaccessibility of carotenoids. Besides the release of certain individual phenolic acids from their complex compounds. During storage, there was a decline in AA in the different samples, and F1 was the lowest. This loss came back to destroy vitamin $\mathrm{C}$, polyphenols, and carotenoids. F4 showed maximal AA\% (36.92 \pm 0.73$)$ at storage ending.

\section{Identification of phenolic compounds by HPLC}

Table 3 summarizes the findings of quantified and separate phenols of cantaloupe juice analyzed by HPLC. The TP in fresh sample was $139.01 \pm 0.14 \mathrm{mg} / 100 \mathrm{~g}$ as gallic acid. The flavonoids (Flavan3-ols) group, which comprises catechin, epicatechin, epicatechin gallate, has the highest percentage of TP with a quantity of up to $60.77 \mathrm{mg} / 100 \mathrm{~g}$

Table 3: Impact of various pasteurized temperatures and storage on the concentration of phenolic compounds identified in different treatments of cantaloupe juice

\begin{tabular}{|c|c|c|c|c|c|c|c|c|c|c|}
\hline \multirow{2}{*}{ Identification } & \multirow[b]{2}{*}{$\mathbf{R t}$} & & \multicolumn{2}{|c|}{ F1 } & \multicolumn{2}{|c|}{ F2 } & \multicolumn{2}{|c|}{ F3 } & \multicolumn{2}{|c|}{ F4 } \\
\hline & & Fresh & 0 time & 4 week & o time & 4 week & 0 time & 4 week & 0 time & 4 week \\
\hline \multicolumn{11}{|c|}{ mg/100g (fresh weight) } \\
\hline \multicolumn{11}{|c|}{ Hydroxybenzoic acid derivatives } \\
\hline Pyrogallic & 2.08 & $7.63 \pm 0.05^{\mathrm{a}}$ & $6.88 \pm 0.08^{\mathrm{b}}$ & $4.81 \pm 0.02^{\mathrm{e}}$ & $6.96 \pm 0.62^{\mathrm{b}}$ & $5.29 \pm 0.06^{\mathrm{d}}$ & $7.37 \pm 0.08^{\mathrm{a}}$ & $5.45 \pm 0.21^{\mathrm{d}}$ & $7.50 \pm 0.12^{\mathrm{a}}$ & $5.86 \pm 0.12^{\mathrm{c}}$ \\
\hline Gallic & 2.18 & $9.32 \pm 0.07^{\mathrm{a}}$ & $8.39 \pm 0.05^{\mathrm{c}}$ & $5.88 \pm 0.06^{f}$ & $8.50 \pm 0.28^{c}$ & $6.46 \pm 0.23^{\mathrm{e}}$ & $8.99 \pm 0.04^{\mathrm{b}}$ & $6.65 \pm 0.18^{\mathrm{e}}$ & $9.16 \pm 0.13^{\mathrm{ab}}$ & $7.15 \pm 0.15^{\mathrm{d}}$ \\
\hline $\begin{array}{l}\text { P OH Benzoic-3-o- } \\
\text { glucoside }\end{array}$ & 3.63 & $3.37 \pm 0.05^{\mathrm{a}}$ & $3.04 \pm 0.08^{b}$ & $2.13 \pm 0.05^{\mathrm{e}}$ & $3.08 \pm 0.05^{\mathrm{b}}$ & $2.34 \pm 0.05^{\mathrm{cd}}$ & $3.25 \pm 0.08^{\mathrm{ab}}$ & $2.41 \pm 0.03^{\mathrm{cd}}$ & $3.31 \pm 0.08^{\mathrm{a}}$ & $2.59 \pm 0.33^{\mathrm{c}}$ \\
\hline P OH Benzoic acid & 8.40 & $2.32 \pm 0.07^{\mathrm{a}}$ & $2.09 \pm 0.13^{\mathrm{abc}}$ & $1.46 \pm 0.04^{\mathrm{d}}$ & $2.12 \pm 0.54^{\mathrm{ab}}$ & $1.61 \pm 0.07^{\mathrm{d}}$ & $2.24 \pm 0.20^{\mathrm{a}}$ & $1.66 \pm 0.06^{\mathrm{cd}}$ & $2.28 \pm 0.25^{\mathrm{a}}$ & $1.78 \pm 0.34^{\mathrm{bcd}}$ \\
\hline \multicolumn{11}{|c|}{ Flavan 3-ols } \\
\hline Catechin & 2.82 & $23.95 \pm 0.04^{\mathrm{a}}$ & $21.57 \pm 0.21^{\mathrm{d}}$ & $15.10 \pm 0.05^{\mathrm{h}}$ & $21.85 \pm 0.52^{\mathrm{d}}$ & $16.61 \pm 0.12^{\mathrm{g}}$ & $23.11 \pm 0.24^{\mathrm{c}}$ & $17.10 \pm 0.06^{f}$ & $23.54 \pm 0.21^{\mathrm{b}}$ & $18.37 \pm 0.24^{\mathrm{e}}$ \\
\hline Epi-catechin & 2.96 & $49.61 \pm 0.25^{\mathrm{a}}$ & $44.70 \pm 0.16^{\mathrm{e}}$ & $31.28 \pm 0.04^{\mathrm{i}}$ & $45.27 \pm 0.15^{\mathrm{d}}$ & $34.40 \pm 0.07^{\mathrm{h}}$ & $47.88 \pm 0.05^{\mathrm{c}}$ & $35.43 \pm 0.08 \mathrm{~g}$ & $48.76 \pm 0.37^{\mathrm{b}}$ & $38.06 \pm 0.34^{\mathrm{f}}$ \\
\hline
\end{tabular}




\begin{tabular}{|c|c|c|c|c|c|c|c|c|c|c|}
\hline Epi-catechin gallate & 3.19 & $3.53 \pm 0.07^{\mathrm{a}}$ & $3.18 \pm 0.11^{\mathrm{b}}$ & $2.23 \pm 0.06^{\mathrm{e}}$ & $3.22 \pm 0.06^{\mathrm{b}}$ & $2.45 \pm 0.08^{\mathrm{d}}$ & $3.41 \pm 0.06^{\mathrm{a}}$ & $2.52 \pm 0.05^{\mathrm{d}}$ & $3.47 \pm 0.06^{\mathrm{a}}$ & $2.71 \pm 0.21^{\mathrm{c}}$ \\
\hline \multicolumn{11}{|c|}{ Hydroxycinnamic acids } \\
\hline Protocatchuic & 2.43 & $3.31 \pm 0.12^{\mathrm{a}}$ & $2.98 \pm 0.04^{\mathrm{b}}$ & $2.09 \pm 0.18^{\mathrm{d}}$ & $3.02 \pm 0.19^{\mathrm{ab}}$ & $2.29 \pm 0.31^{\mathrm{cd}}$ & $3.19 \pm 0.17^{\mathrm{ab}}$ & $2.36 \pm 0.05^{\mathrm{cd}}$ & $3.25 \pm 0.05^{\mathrm{ab}}$ & $2.54 \pm 0.08^{\mathrm{c}}$ \\
\hline Chlorogenic acid & 2.61 & $8.17 \pm 0.02^{\mathrm{a}}$ & $7.36 \pm 0.34^{\mathrm{b}}$ & $5.15 \pm 0.07^{\mathrm{e}}$ & $7.46 \pm 0.13^{\mathrm{b}}$ & $5.67 \pm 0.17^{\mathrm{d}}$ & $7.89 \pm 0.34^{\mathrm{a}}$ & $5.84 \pm 0.14^{\mathrm{d}}$ & $8.03 \pm 0.14^{\mathrm{a}}$ & $6.27 \pm 0.07^{\mathrm{c}}$ \\
\hline Caffeic acid & 3.91 & $0.57 \pm 0.06^{\mathrm{a}}$ & $0.52 \pm 0.02^{\mathrm{ab}}$ & $0.36 \pm 0.12^{\mathrm{c}}$ & $0.52 \pm 0.08^{\mathrm{ab}}$ & $0.40 \pm 0.04^{\mathrm{bc}}$ & $0.55 \pm 0.13^{\mathrm{a}}$ & $0.41 \pm 0.04^{\mathrm{bc}}$ & $0.56 \pm 0.09^{\mathrm{a}}$ & $0.44 \pm 0.02^{\mathrm{abc}}$ \\
\hline Syringic acid & 4.11 & $0.16 \pm 0.01^{\mathrm{a}}$ & $0.14 \pm 0.07^{\mathrm{a}}$ & $0.10 \pm 0.03^{\mathrm{a}}$ & $0.15 \pm 0.12^{\mathrm{a}}$ & $0.11 \pm 0.09^{\mathrm{a}}$ & $0.15 \pm 0.12^{\mathrm{a}}$ & $0.11 \pm 0.03^{\mathrm{a}}$ & $0.16 \pm 0.04^{\mathrm{a}}$ & $0.12 \pm 0.06^{\mathrm{a}}$ \\
\hline Vanillic acid & 4.73 & $6.20 \pm 0.05^{\mathrm{a}}$ & $5.59 \pm 0.04^{\mathrm{c}}$ & $3.91 \pm 0.08^{\mathrm{f}}$ & $5.66 \pm 0.33^{\mathrm{bc}}$ & $4.30 \pm 0.11^{\mathrm{e}}$ & $5.99 \pm 0.26^{\mathrm{ab}}$ & $4.43 \pm 0.06^{\mathrm{de}}$ & $6.09 \pm 0.18^{\mathrm{a}}$ & $4.76 \pm 0.42^{\mathrm{d}}$ \\
\hline P-coumaric acid & 5.98 & $2.99 \pm 0.06^{\mathrm{a}}$ & $2.69 \pm 0.05^{\mathrm{b}}$ & $1.89 \pm 0.07^{\mathrm{d}}$ & $2.73 \pm 0.17^{\mathrm{ab}}$ & $2.07 \pm 0.12^{\mathrm{cd}}$ & $2.89 \pm 0.06^{\mathrm{ab}}$ & $2.13 \pm 0.19^{\mathrm{cd}}$ & $2.94 \pm 0.23^{\mathrm{ab}}$ & $2.29 \pm 0.27^{\mathrm{c}}$ \\
\hline Ferrulic acid & 6.14 & $0.76 \pm 0.14^{\mathrm{a}}$ & $0.69 \pm 0.03^{\mathrm{a}}$ & $0.48 \pm 0.14^{\mathrm{a}}$ & $0.70 \pm 0.20^{\mathrm{a}}$ & $0.53 \pm 0.03^{\mathrm{a}}$ & $0.74 \pm 0.23^{\mathrm{a}}$ & $0.55 \pm 0.05^{\mathrm{a}}$ & $0.75 \pm 0.32^{\mathrm{a}}$ & $0.59 \pm 0.13^{\mathrm{a}}$ \\
\hline Iso ferulic & 6.22 & $0.41 \pm 0.11^{\mathrm{a}}$ & $0.37 \pm 0.06^{\mathrm{a}}$ & $0.26 \pm 0.09^{\mathrm{a}}$ & $0.38 \pm 0.06^{\mathrm{a}}$ & $0.29 \pm 0.06^{\mathrm{a}}$ & $0.40 \pm 0.04^{\mathrm{a}}$ & $0.30 \pm 0.07^{\mathrm{a}}$ & $0.41 \pm 0.17^{\mathrm{a}}$ & $0.32 \pm 0.18^{\mathrm{a}}$ \\
\hline E- vanillic & 8.12 & $0.16 \pm 0.04^{\mathrm{a}}$ & $0.14 \pm 0.09^{\mathrm{a}}$ & $0.10 \pm 0.07^{\mathrm{a}}$ & $0.15 \pm 0.07^{\mathrm{a}}$ & $0.11 \pm 0.04^{\mathrm{a}}$ & $0.15 \pm 0.07^{\mathrm{a}}$ & $0.11 \pm 0.05^{\mathrm{a}}$ & $0.16 \pm 0.03^{\mathrm{a}}$ & $0.12 \pm 0.06^{\mathrm{a}}$ \\
\hline O-coumaric & 8.24 & $3.05 \pm 0.06^{\mathrm{a}}$ & $2.75 \pm 0.11^{\mathrm{ab}}$ & $1.93 \pm 0.05^{\mathrm{c}}$ & $2.79 \pm 0.46^{\mathrm{ab}}$ & $2.12 \pm 0.06^{\mathrm{c}}$ & $2.95 \pm 0.33^{\mathrm{a}}$ & $2.18 \pm 0.47^{\mathrm{c}}$ & $3.00 \pm 0.05^{\mathrm{a}}$ & $2.34 \pm 0.09^{\mathrm{bc}}$ \\
\hline Cinnamic acid & 12.55 & $3.94 \pm 0.15^{\mathrm{a}}$ & $3.55 \pm 0.08^{\mathrm{a}}$ & $2.49 \pm 0.06^{\mathrm{c}}$ & $3.60 \pm 0.15^{\mathrm{a}}$ & $2.73 \pm 0.18^{\mathrm{bc}}$ & $3.81 \pm 0.63^{\mathrm{a}}$ & $2.82 \pm 0.04^{\mathrm{bc}}$ & $3.88 \pm 0.16^{\mathrm{a}}$ & $3.03 \pm 0.12^{\mathrm{b}}$ \\
\hline \multicolumn{11}{|c|}{ Hydrolyzed tannins } \\
\hline Pyrogallol & 2.29 & $9.45 \pm 0.13^{\mathrm{a}}$ & $8.51 \pm 0.03^{\mathrm{c}}$ & $5.96 \pm 0.01^{\mathrm{f}}$ & $8.62 \pm 0.21^{\mathrm{c}}$ & $6.55 \pm 0.27^{\mathrm{e}}$ & $9.12 \pm 0.03^{\mathrm{b}}$ & $6.75 \pm 0.03^{\mathrm{e}}$ & $9.28 \pm 0.08^{\mathrm{ab}}$ & $7.25 \pm 0.14^{\mathrm{d}}$ \\
\hline \multicolumn{11}{|c|}{ Ellagic acid and derivatives } \\
\hline Ellagic acid & 6.75 & $0.10 \pm 0.02^{\mathrm{a}}$ & $0.09 \pm 0.05^{\mathrm{a}}$ & $0.06 \pm 0.11^{\mathrm{a}}$ & $0.09 \pm 0.04^{\mathrm{a}}$ & $0.07 \pm 0.02^{\mathrm{a}}$ & $0.09 \pm 0.03^{\mathrm{a}}$ & $0.07 \pm 0.04^{\mathrm{a}}$ & $0.09 \pm 0.01^{\mathrm{a}}$ & $0.07 \pm 0.05^{\mathrm{a}}$ \\
\hline Total & & $139.01 \pm 0.14$ & $125.23 \pm 0.0$ & $87.66 \pm 0.08$ & $126.85 \pm 0.22$ & $96.40 \pm 0.13$ & $134.16 \pm 0.1$ & $99.27 \pm 0.11$ & $136.62 \pm 0.1$ & $106.65 \pm 0.17$ \\
\hline
\end{tabular}

The values are the mean \pm margin of confidence intervals at $95 \%$.

Data in the same row with different letters differ significantly $(p<0.05)$.

of $139.01 \pm 0.14 \mathrm{mg} / 100 \mathrm{~g}$, with a level of $43.71 \%$ of TP in a fresh sample. The hydroxybenzoic acid group plus the hydroxycinnamic group represent the phenolic acids in cantaloupe juice. The hydroxycinnamic acid group is composed of a of $21.37 \%$ of TP, containing compounds such as, protocatechuic acid, chlorogenic acid, caffeic acid, syringic acid, vanilla acid, P-coumaric acid, ferulic acid, isoferulic acid, E-vanillic acid, O-coumaric acid, and cinnamic acid. The hydroxybenzoic acid group presented by $10.79 \%$ of TP, which contained gallic acid, P-hydroxy benzoic-3-O-glucoside, and P-hydroxy benzoic acid. The hydrolyzed group of tannins, which comprises pyrogallic and pyrogallol consists of $12.28 \%$ from TP in fresh sample. The ellagic acid group derivatives include ellagic acid alone, which accounted for $0.072 \%$ of TP in a fresh sample. The results showed that the TP content decreased to $125.23 \pm 0.09$ with a loss rate of $1.71 \%-9.86 \%$ during the pasteurization process. The results also reveal that pasteurization at $90^{\circ} \mathrm{C} / 45$ seconds had kept on TP compared to pasteurized at $85^{\circ} \mathrm{C}$ for 90 seconds. The drop in TP continued over 28 days of storing. Samples kept at room temperature recorded a loss rate of TP between (31.34 and $37 \%$ ) compared to (23.27 to $28.58 \%$ ) for samples stored at $4{ }^{\circ} \mathrm{C}$. Based on these results, the refrigerated juice had kept a higher amount of TP than stored at room temperature by percent range (8.07 to $8.42 \%)$. The rate loss of (T1, T2, T3, and T4) were $(36.93,30.65,28.58$, and $23.27 \%)$ at the end of storage.

\section{Color parameter of pasteurized cantaloupe juice during storage}

The findings showed in Table 4 is regarding the color values L, a, b, Hue, and chroma for cantaloupe juice. The thermal treatments showed a significant decrease in $\mathrm{L}^{*}$ values after heat processing, especially in samples pasteurized at $85^{\circ} \mathrm{C}$ such as F1, F2 (76.18 \pm 0.73 and $76.08 \pm 0.11)$ while, F4 was the greatest $L^{*}(77.30 \pm 0.18)$. There has been a significant decrease in the $\mathrm{L}^{*}$ value of samples stored at room temperature than refrigerated samples. F4 recorded the highest $\mathrm{L}^{*}$ value $(75.13 \pm 0.21)$, followed by $\mathrm{F} 2$ with a value of (74.67 \pm 0.13$)$ while, F1 was the lowest treatment at the storage ending. Concerning a * value, both treatments have shown that $\mathrm{a} *$ is lower than the fresh sample. Pasteurization at 90 ${ }^{\circ} \mathrm{C} / 45$ seconds, preserved $\mathrm{a}^{*}$ compared to $85{ }^{\circ} \mathrm{C} / 90$ seconds due to a lack of carotenoid degradation that responds to yellow, orange, and red. When the storage effects comparing,

Table 4: Impact of various pasteurized temperatures and storage on $\mathrm{L}^{*}, \mathrm{a}^{*}, \mathrm{~b}^{*}$, Hue and Chroma of cantaloupe juice

\begin{tabular}{|c|c|c|c|c|c|c|}
\hline Sample & Storage weeks & $\mathbf{L}^{*}$ & $a^{*}$ & $\mathbf{b}^{*}$ & Heu & Chroma \\
\hline $\mathbf{F}$ & Fresh & $79.17 \pm 0.07^{a}$ & $2.34 \pm 0.08^{a}$ & $5.44 \pm 0.15^{\mathrm{a}}$ & $66.73 \pm 0.14^{\mathrm{m}}$ & $5.92 \pm 0.17^{\mathrm{a}}$ \\
\hline \multirow{5}{*}{ F1 } & 0 time & $76.18 \pm 0.73^{\mathrm{de}}$ & $1.36 \pm 0.08^{\mathrm{bc}}$ & $3.91 \pm 0.4^{\mathrm{bc}}$ & $70.82 \pm 0.86^{\mathrm{jkl}}$ & $4.14 \pm 0.06^{\mathrm{bc}}$ \\
\hline & 1week & $74.87 \pm 0.56^{\mathrm{hi}}$ & $1.19 \pm 0.05^{\mathrm{de}}$ & $3.65 \pm 0.05^{\mathrm{d}}$ & $71.94 \pm 0.48^{\text {hijk }}$ & $3.84 \pm 0.06^{\mathrm{ef}}$ \\
\hline & 2week & $73.71 \pm 0.40^{\mathrm{jk}}$ & $0.94 \pm 0.09^{\text {ghi }}$ & $3.33 \pm 0.06^{\mathrm{ef}}$ & $74.24 \pm 1.16^{\mathrm{defg}}$ & $3.46 \pm 0.08^{\mathrm{g}}$ \\
\hline & 3 Week & $72.83 \pm 0.34^{1}$ & $0.71 \pm 0.05^{\mathrm{kl}}$ & $2.97 \pm 0.10^{\mathrm{ij}}$ & $76.56 \pm 0.48^{\mathrm{bc}}$ & $3.05 \pm 0.11 \mathrm{j}^{\mathrm{k}}$ \\
\hline & 4week & $71.45 \pm 0.16^{\mathrm{m}}$ & $0.48 \pm 0.06^{\mathrm{m}}$ & $2.65 \pm 0.08^{\mathrm{k}}$ & $79.73 \pm 0.95^{\mathrm{a}}$ & $2.69 \pm 0.09^{1}$ \\
\hline \multirow{5}{*}{$\mathrm{F} 2$} & 0 time & $76.08 \pm 0.11^{\mathrm{de}}$ & $1.38 \pm 0.05^{\mathrm{b}}$ & $3.93 \pm 0.09^{\mathrm{bc}}$ & $70.65 \pm 0.24^{\mathrm{jkl}}$ & $4.17 \pm 0.10^{\mathrm{bc}}$ \\
\hline & 1week & $75.86 \pm 0.08^{\mathrm{ef}}$ & $1.19 \pm 0.08^{\mathrm{de}}$ & $3.72 \pm 0.05^{\mathrm{cd}}$ & $72.26 \pm 0.89^{\text {ghij }}$ & $3.91 \pm 0.07^{\mathrm{df}}$ \\
\hline & 2 week & $75.29 \pm 0.07^{\text {fghi }}$ & $1.02 \pm 0.03^{\mathrm{fg}}$ & $3.54 \pm 0.06^{\mathrm{de}}$ & $73.93 \pm 0.19^{\text {efgh }}$ & $3.68 \pm 0.07^{\mathrm{f}}$ \\
\hline & 3 Week & $74.95 \pm 0.16^{\mathrm{hi}}$ & $0.88 \pm 0.11^{\text {ghij }}$ & $3.29 \pm 0.04^{\mathrm{fg}}$ & $75.03 \pm 1.61^{\text {cdef }}$ & $3.41 \pm 0.07^{\mathrm{gh}}$ \\
\hline & 4week & $74.67 \pm 0.13^{\mathrm{i}}$ & $0.76 \pm 0.05^{\mathrm{jkl}}$ & $3.08 \pm 0.08^{\text {ghij }}$ & $76.14 \pm 0.53^{\mathrm{bcd}}$ & $3.17 \pm 0.09^{\mathrm{ij}}$ \\
\hline \multirow{5}{*}{ F3 } & 0 time & $77.14 \pm 0.55^{\mathrm{bc}}$ & $1.52 \pm 0.06^{\mathrm{b}}$ & $3.96 \pm 0.05^{\mathrm{b}}$ & $69.00 \pm 0.511$ & $4.24 \pm 0.07^{\mathrm{bc}}$ \\
\hline & 1week & $76.67 \pm 0.64^{\mathrm{cd}}$ & $1.21 \pm 0.08^{\mathrm{cd}}$ & $3.61 \pm 0.09^{\mathrm{d}}$ & $71.47 \pm 0.43^{\mathrm{ijk}}$ & $3.81 \pm 0.10^{\mathrm{ef}}$ \\
\hline & 2 week & $75.54 \pm 0.47^{\text {efgh }}$ & $0.99 \pm 0.11^{\text {fgh }}$ & $3.27 \pm 0.06^{\mathrm{fgh}}$ & $73.16 \pm 1.47^{\text {fghi }}$ & $3.42 \pm 0.09^{\mathrm{gh}}$ \\
\hline & 3Week & $74.03 \pm 0.24^{j}$ & $0.78 \pm 0.13^{\mathrm{ijkl}}$ & $3.06 \pm 0.10^{\text {hij }}$ & $75.70 \pm 1.84^{\text {bcde }}$ & $3.16 \pm 0.13^{\mathrm{ij}}$ \\
\hline & 4week & $73.15 \pm 0.13^{\mathrm{kl}}$ & $0.63 \pm 0.07^{1 \mathrm{~m}}$ & $2.89 \pm 0.07^{j}$ & $77.70 \pm 1.04^{\mathrm{b}}$ & $2.96 \pm 0.08^{\mathrm{k}}$ \\
\hline \multirow{4}{*}{ F4 } & 0 time & $77.30 \pm 0.18^{b}$ & $1.48 \pm 0.13^{\mathrm{b}}$ & $4.04 \pm 0.14^{\mathrm{b}}$ & $69.88 \pm 0.99^{\mathrm{kl}}$ & $4.30 \pm 0.18^{b}$ \\
\hline & 1week & $76.08 \pm 0.22^{\mathrm{de}}$ & $1.13 \pm 0.07^{\mathrm{def}}$ & $3.91 \pm 0.04^{\mathrm{bc}}$ & $73.88 \pm 0.79^{\text {efgh }}$ & $4.07 \pm 0.06^{\mathrm{cd}}$ \\
\hline & 2 week & $75.68 \pm 0.60^{\text {efg }}$ & $1.03 \pm 0.06^{\mathrm{efg}}$ & $3.53 \pm 0.12^{\mathrm{de}}$ & $73.73 \pm 0.37^{\text {efgh }}$ & $3.68 \pm 0.13^{f}$ \\
\hline & 3 Week & $75.34 \pm 0.16^{\text {fghi }}$ & $0.97 \pm 0.05^{\text {fgh }}$ & $3.33 \pm 0.08^{\mathrm{ef}}$ & $73.76 \pm 0.42^{\text {efgh }}$ & $3.47 \pm 0.09^{\mathrm{g}}$ \\
\hline
\end{tabular}




\begin{tabular}{l|l|l} 
4week & $75.13 \pm 0.21^{\text {ghi }}$ & $0.83 \pm 0.22^{\text {hijk }}$
\end{tabular}

The values are the mean \pm margin of confidence intervals at $95 \%$.

Data in the same column with different letters differ significantly $(p<0.05)$.

$\mathrm{a}^{*}$ value $(\mathrm{F} 2, \mathrm{~F} 4)$ of the samples stored at $4{ }^{\circ} \mathrm{C}$ remained higher than $(\mathrm{F} 1, \mathrm{~F} 3) . \mathrm{F} 4$ with a range of $(0.83 \pm 0.22)$ had the maximal value of $\mathrm{a}^{*}$. The fresh sample was more representing to yellow (66.73) due to the higher content of carotenoids. The increased hue value (77) due to the destruction of the carotenoids by processing and storage made colorapproaching green-yellow. F4 held the lowest hue (75.24 \pm 3,52 ) in contrast to other treatments by storage end. The same pattern of decline in $\mathrm{L}, \mathrm{a}, \mathrm{b}$, and hue realized by chroma (color clarity). After processing, color clarity decreased, and the sequence of treatments is in terms of preference $(\mathrm{F} 4, \mathrm{~F} 3$, $\mathrm{F} 2$, and F1). A higher temperature for a short time $\left(90^{\circ} \mathrm{C} / 45 \mathrm{sec}\right)$ was better than the lower temperature for a long time $\left(85^{\circ} \mathrm{C} / 90 \mathrm{sec}\right)$. The order of sample preferences by storage end was (F4, F2, F3, and F1). The previous results declared that the cold storage of cantaloupe juice pasteurized for a short time $\left(90^{\circ} \mathrm{C} / 45 \mathrm{sec}\right)$ was the desired.

\section{Sensory attributes of pasteurized cantaloupe juice during storage}

Table 5 displays the scores of various sensory qualities for cantaloupe juice at zero time and after storage. Comparing samples after processing with fresh samples revealed a substantial decline in all sensory attributes such as taste, color, odor, appearance, mouthfeel, and overall acceptability. No major differences were identified between the three treatments $(\mathrm{F} 4, \mathrm{~F} 3, \mathrm{~F} 2)$ at zero time. During storage, there was a substantial decline in all sensory attributes of the different treatments under study. F4 was the best treatment with maximum overall acceptability $(7.41 \pm 0.04)$ followed by $F 2$, both of which were kept in cold conditions.

\section{Discussion}

The results suggest that the temperatures used for pasteurization did not contribute to changes in TSS content at the start of storage. TSS differences after storage were marginally minor and pasteurization at $90{ }^{\circ} \mathrm{C} / 45 \mathrm{sec}$ with refrigerated storage reported the lowest changes in TSS.

As for $\mathrm{pH}$, no variations were found between treatments after adjusted $\mathrm{pH}$ and thermal treated at zero time. Also, storage temperature did not influence the $\mathrm{pH}$ because there were no undesirable changes compared to the samples at zero time. No effects of various treatments on the acidity of cantaloupe juice were also found at the start or end of storage after 28 days of storage.

Vitamin $\mathrm{C}$ is considered an indicator of the nutritional values of fruit juices (Rabie et al., 2015) ${ }^{[24]}$.

Table 5: Impact of various pasteurized temperatures and storage on taste, color, odor, appearance, mouthful, and overall acceptability of cantaloupe juice

\begin{tabular}{|c|c|c|c|c|c|c|c|}
\hline Sample & Storage weeks & Taste (9) & Color (9) & Odor (9) & Appearance (9) & Mouthfeel (9) & Overall acceptability (9) \\
\hline $\mathrm{F}$ & Fresh & $8.43 \pm 0.03^{\mathrm{a}}$ & $9.00 \pm 0.00^{\mathrm{a}}$ & $8.68 \pm 0.06^{\mathrm{a}}$ & $8.64 \pm 0.22^{\mathrm{a}}$ & $8.72 \pm 0.03^{\mathrm{a}}$ & $8.69 \pm 0.07^{\mathrm{a}}$ \\
\hline \multirow[t]{5}{*}{ F1 } & 0 time & $7.96 \pm 0.09^{\mathrm{cd}}$ & $8.05 \pm 0.07^{\mathrm{hi}}$ & $7.83 \pm 0.07^{\mathrm{e}}$ & $7.89 \pm 0.05^{\mathrm{ef}}$ & $8.14 \pm 0.06^{\mathrm{bc}}$ & $7.97 \pm 0.07^{\mathrm{d}}$ \\
\hline & 1week & $7.73 \pm 0.05^{\mathrm{fg}}$ & $7.93 \pm 0.04^{\mathrm{ij}}$ & $7.63 \pm 0.11^{\mathrm{f}}$ & $7.70 \pm 0.04^{\mathrm{gh}}$ & $7.73 \pm 0.05^{\mathrm{e}}$ & $7.74 \pm 0.06^{\mathrm{ef}}$ \\
\hline & 2 week & $7.39 \pm 0.04^{\mathrm{i}}$ & $7.81 \pm 0.12^{\mathrm{jk}}$ & $7.42 \pm 0.02^{\mathrm{gh}}$ & $7.43 \pm 0.13^{\mathrm{i}}$ & $7.28 \pm 0.03^{f}$ & $7.47 \pm 0.07^{\mathrm{g}}$ \\
\hline & 3 Week & $7.17 \pm 0.13^{\mathrm{jk}}$ & $7.68 \pm 0.16^{\mathrm{kl}}$ & $7.19 \pm 0.06^{\mathrm{ij}}$ & $7.19 \pm 0.15^{\mathrm{j}}$ & $6.91 \pm 0.06^{\mathrm{g}}$ & $7.23 \pm 0.11^{\mathrm{h}}$ \\
\hline & 4week & $6.82 \pm 0.06^{1}$ & $7.52 \pm 0.18^{1}$ & $6.92 \pm 0.07^{\mathrm{k}}$ & $6.90 \pm 0.08^{\mathrm{k}}$ & $6.53 \pm 0.07^{\mathrm{h}}$ & $6.94 \pm 0.09^{j}$ \\
\hline \multirow{5}{*}{$\mathrm{F} 2$} & 0 time & $8.03 \pm 0.04^{\mathrm{bc}}$ & $8.44 \pm 0.13^{\mathrm{cd}}$ & $8.02 \pm 0.04^{\mathrm{cd}}$ & $8.23 \pm 0.05^{\mathrm{bc}}$ & $8.03 \pm 0.12^{\mathrm{cd}}$ & $8.15 \pm 0.08^{c}$ \\
\hline & 1week & $7.76 \pm 0.05^{\mathrm{fg}}$ & $8.35 \pm 0.08^{\mathrm{cde}}$ & $7.90 \pm 0.14^{\mathrm{de}}$ & $8.05 \pm 0.05^{\mathrm{de}}$ & $7.74 \pm 0.08^{\mathrm{e}}$ & $7.96 \pm 0.08^{\mathrm{d}}$ \\
\hline & 2 week & $7.61 \pm 0.07^{\mathrm{h}}$ & $8.24 \pm 0.07^{\text {efgh }}$ & $7.66 \pm 0.12^{\mathrm{f}}$ & $7.68 \pm 0.04^{\mathrm{gh}}$ & $7.38 \pm 0.07^{\mathrm{f}}$ & $7.71 \pm 0.07^{\mathrm{f}}$ \\
\hline & 3Week & $7.28 \pm 0.04^{j}$ & $8.15 \pm 0.06^{\text {fgh }}$ & $7.40 \pm 0.07^{\mathrm{h}}$ & $7.36 \pm 0.08^{\mathrm{i}}$ & $6.92 \pm 0.6^{\mathrm{g}}$ & $7.42 \pm 0.06^{\mathrm{g}}$ \\
\hline & 4week & $7.11 \pm 0.07^{\mathrm{k}}$ & $7.93 \pm 0.03^{\mathrm{ij}}$ & $7.22 \pm 0.17^{\mathrm{ij}}$ & $7.14 \pm 0.08^{j}$ & $6.56 \pm 0.02^{\mathrm{h}}$ & $7.19 \pm 0.07^{\mathrm{hi}}$ \\
\hline \multirow{5}{*}{ F3 } & 0 time & $8.07 \pm 0.03^{\mathrm{b}}$ & $8.52 \pm 0.21^{\mathrm{bc}}$ & $8.14 \pm 0.13^{\mathrm{c}}$ & $8.27 \pm 0.04^{b}$ & $8.09 \pm 0.12^{\mathrm{c}}$ & $8.22 \pm 0.11^{\mathrm{c}}$ \\
\hline & 1week & $7.83 \pm 0.02^{\mathrm{ef}}$ & $8.31 \pm 0.16^{\text {defg }}$ & $7.44 \pm 0.08^{\mathrm{gh}}$ & $7.94 \pm 0.14^{\mathrm{def}}$ & $7.79 \pm 0.04^{\mathrm{de}}$ & $7.86 \pm 0.09^{\mathrm{de}}$ \\
\hline & 2 week & $7.61 \pm 0.06^{\mathrm{h}}$ & $8.14 \pm 0.14^{\mathrm{gh}}$ & $7.67 \pm 0.04^{\mathrm{f}}$ & $7.62 \pm 0.03^{\mathrm{h}}$ & $7.33 \pm 0.02^{\mathrm{f}}$ & $7.67 \pm 0.06^{\mathrm{f}}$ \\
\hline & 3Week & $7.26 \pm 0.11^{\mathrm{j}}$ & $7.92 \pm 0.08^{\mathrm{ij}}$ & $7.31 \pm 0.03^{\text {hi }}$ & $7.36 \pm 0.02^{\mathrm{i}}$ & $6.82 \pm 0.10^{\mathrm{g}}$ & $7.33 \pm 0.07^{\mathrm{gh}}$ \\
\hline & 4week & $6.92 \pm 0.05^{1}$ & $7.71 \pm 0.09^{\mathrm{k}}$ & $7.14 \pm 0.15^{j}$ & $7.07 \pm 0.06^{j}$ & $6.48 \pm 0.15^{\mathrm{h}}$ & $7.06 \pm 0.10^{\mathrm{ij}}$ \\
\hline \multirow{5}{*}{$\mathrm{F} 4$} & 0 time & $8.11 \pm 0.05^{\mathrm{b}}$ & $8.63 \pm 0.04^{\mathrm{b}}$ & $8.35 \pm 0.07^{\mathrm{b}}$ & $8.33 \pm 0.18^{b}$ & $8.36 \pm 0.04^{\mathrm{b}}$ & $8.36 \pm 0.08^{b}$ \\
\hline & 1week & $7.92 \pm 0.01^{\mathrm{de}}$ & $8.34 \pm 0.04^{\text {cdef }}$ & $8.17 \pm 0.05^{\mathrm{c}}$ & $8.08 \pm 0.12^{\mathrm{cd}}$ & $8.12 \pm 0.12^{\mathrm{bc}}$ & $8.13 \pm 0.07^{\mathrm{c}}$ \\
\hline & 2 week & $7.66 \pm 0.08^{\mathrm{gh}}$ & $8.30 \pm 0.02^{\text {defg }}$ & $8.04 \pm 0.09^{\mathrm{cd}}$ & $7.80 \pm 0.03^{\mathrm{fg}}$ & $7.70 \pm 0.05^{\mathrm{e}}$ & $7.90 \pm 0.05^{\mathrm{d}}$ \\
\hline & 3Week & $7.47 \pm 0.04^{\mathrm{i}}$ & $8.17 \pm 0.03^{\text {efgh }}$ & $7.89 \pm 0.02^{\mathrm{de}}$ & $7.68 \pm 0.05^{\mathrm{gh}}$ & $7.35 \pm 0.06^{\mathrm{f}}$ & $7.71 \pm 0.04^{\mathrm{f}}$ \\
\hline & 4week & $7.19 \pm 0.06^{\mathrm{j}^{\mathrm{k}}}$ & $8.05 \pm 0.05^{\mathrm{hi}}$ & $7.56 \pm 0.03^{\mathrm{fg}}$ & $7.43 \pm 0.02^{\mathrm{i}}$ & $6.83 \pm 0.02^{\mathrm{g}}$ & $7.41 \pm 0.04^{\mathrm{g}}$ \\
\hline
\end{tabular}

The values are the mean \pm margin of confidence intervals at $95 \%$.

Data in the same column with different letters differ significantly $(p<0.05)$

Vitamin $\mathrm{C}$ is one of the important bioactive compounds affected by the level of temperature and industrial practices during processing. The temperature used for pasteurization decreased vitamin $\mathrm{C}$ after processing compared to the fresh sample. Increasing the degree of pasteurization reduced the vitamin $\mathrm{C}$ content compared with lower temperature. However, the higher degree $\left(90^{\circ} \mathrm{C}\right)$ maintained the vitamin eventually of storage. The storage temperature was a significant effect on the residual vitamin, and the cold storage retained a higher amount of vitamin $\mathrm{C}$. VC content decreased dramatically during storage. This decline was due to the dissolved oxygen in the headspace in the early stages of storage, which oxidized ascorbic acid in the juice or by light exposure (Embaby and Mokhtar, 2019) ${ }^{[7]}$.

A strong correlation was found between the higher vitamin $\mathrm{C}$ content and the reduction of browning in the samples by storage end. The preferred order of samples in terms of preference for vitamin $\mathrm{C}$ retained, as follows (F4, F2, F3, F1) is associated with the trend to decrease browning. During storage, browning increased in cantaloupe juice, due to the 
development of brown pigment over polymerization between ascorbic acid or sugar degradation products or phenolic compounds (Wibowo et al., 2015) ${ }^{[33]}$. A similar decrease in vitamin was found in a refrigerated stored cantaloupe juice stored at $(5 \pm 2 \mathrm{C})$ for 13 days by Sroy et al. (2019) ${ }^{[29]}$. The same previous order in terms of preference for vitamin $\mathrm{C}$ and reduced browning is of the same order obtained by $\mathrm{L}^{*}, \mathrm{a}^{*}, \mathrm{~b}^{*}$, hue, and chroma parameters. A close correlation exists between increasing color quality and vitamin content. Generally, all color parameters $\mathrm{L}^{*}, \mathrm{a}^{*}, \mathrm{~b}^{*}$, hue, chroma were decreased, and browning increased in all samples with storage end. Mokhtar and Ibrahim (2020) ${ }^{[21]}$ have reported related results on guava nectar.

Total phenolic compounds were affected by the degree of pasteurization, the higher degree $\left(90^{\circ} \mathrm{C}\right)$ had less effect, and this compatible with the antioxidant activity at zero time, confirming that the higher degree $\left(90{ }^{\circ} \mathrm{C}\right)$ increased antioxidant activity than $\left(85^{\circ} \mathrm{C}\right)$. This may be due to the release of bound phytochemicals from the insoluble portion after heat treatment, such as carotenoids, by disturbing cell membranes and cell walls, increasing bioaccessible carotenoids, and enhancing absorption (Eberhardt et al., 2000) [6]. The second explanation is related to the impact of pasteurization on conjugate types of phenolic acids, such as chlorogenic acid, which has been converted into caffeic and quinic acid and has improved the antioxidant capacity. By comparing the results of phenols and antioxidant activity at the beginning and end of storage, the correlation between them becomes clear. Generally, total phenolic compounds and antioxidant activity decreased with increased storage period. The pattern of our results was close to that observed by Tahar et al. (2019) ${ }^{[30]}$ in pasteurized pear juice and orange juice. The degradation in vitamins, polyphenols, and carotenoids during storage decreased antioxidant activity in juices (Sroy et al., 2019) [29]. Although cantaloupe juice is not high in phenolic content but contains a higher percentage of Hydroxylated cinnamic acids than Hydroxybenzoic acid derivatives, which are more antioxidant activity than Hydroxybenzoic acid derivatives due to their esters have more hydroxyl in the molecule. Related findings were obtained (Tomas-Barberan and Clifford, 2000; Fu et al., 2011) ${ }^{[31,9]}$.

By comparing the carotenoid findings with the values of $\mathrm{a}^{*}$, $\mathrm{b}^{*}$, and Hue, it has been shown that there is a connection between them. Carotenoids responded to color ranges from yellow to reddish-orange, while the decline in carotenoid content reduced the three parameters. Pasteurization and storage decreased the content of carotenoids, as well as all related parameters, decreased during storage. Similar findings have been conducted by (Sharma et al. 2019) ${ }^{[27]}$ through storage on various forms of juice. The preferred arrangement of treatments due to taste, color, appearance, and overall acceptability was (F4, F2, F3, F1), which the same favored order for samples due to vitamin $\mathrm{C}$ content, browning reduction, $\mathrm{L}^{*}, \mathrm{a}^{*}, \mathrm{~b}^{*}$, hue, and chroma.

These results showed the correlation between sensory attributes and the results of different previous analyses. Generally, all sensory attributes were decreased at storage ending. Present findings are compatible with those of (Ahmed et al., 2019) ${ }^{[1]}$.

The variations in the values of the physicochemical and sensory analysis show that the use of elevated degrees of pasteurization $\left(90^{\circ} \mathrm{C}\right)$ for 45 seconds with refrigerated storage maintains the higher quality of cantaloupe juice after 28 days of storage.

\section{Conclusion}

Heat treatment and storage temperature had significant effects on the bioactive compounds and AA of cantaloupe juice. Heat treatment at 85 and $90^{\circ} \mathrm{C}$ promoted the increase in antioxidant activity of cantaloupe juice. However, the heat treatment at $85^{\circ} \mathrm{C}$ for $90 \mathrm{sec}$ had a marked influence on the sensory, color quality, and bioactive compounds of cantaloupe juice, but heating at $90^{\circ} \mathrm{C} /$ for $45 \mathrm{sec}$ maintained the original quality of cantaloupe juice. The refrigerated storage at $4^{\circ} \mathrm{C}$ maintained all quality parameters compared with storage at ambient temperature. Therefore, pasteurization at $90^{\circ} \mathrm{C} / 45 \mathrm{sec}$ with refrigerated storage could use as an appropriate process for heating and storage cantaloupe juice. The content of TP in cantaloupe juice is well preserved under storage at $4{ }^{\circ} \mathrm{C}$.

\section{References}

1. Ahmed U, Ahmad RS, Mushtaq Z, Hussian SM. Characterization of low calorie ready-to-serve peach beverage using natural sweetener, Stevia (Stevia rebaudiana Bertoni). Journal of nutrition and internal medicine 2019;21(1):435-444.

2. AOAC. Association of Official Analytical Chemists. Official Methods of Analysis of the AOAC Int., 17th Ed. Gaithersburg, Marylad, USA 2000.

3. Banožić M, Babić J, Jokić S. Recent advances in extraction of bioactive compounds from tobacco industrial waste-a review. Industrial Crops and Products 2020;144:112009.

4. Barros L, Cabrita L, Boas MV, Carvalho AM, Ferreira ICFR. Chemical, biochemical and electrochemical assays to evaluate phytochemicals and antioxidant activity of wild plants. Food Chem 2011;127:1600-1608. https://doi.org/10.1016/j.foodchem.2011.02.024

5. Biazotto KR, LM de S, Mesquita BV, Neves ARC, Braga $\mathrm{M}$, Tangerina $\mathrm{W}$ et al. Brazilian biodiversity fruits: discovering bioactive compounds from underexplored sources. Journal of Agricultural and Food Chemistry 2019. Https://doi.org/10.1021/acs. jafc. 8b05815.

6. Eberhardt MV, Lee CY, Liu RH. Antioxidant activity of fresh apples. Nature 2000;405:903-904.

7. Embaby HES, Mokhtar SM. Impact of adding goldenberry (Physalis peruviana L.) on some quality characteristics and bio-functional properties of pasteurized carrot (Daucus carota L.) nectar. Journal of Food Science and Technology 2019;56:966-975.

8. Ferrante A, Incrocci L, Maggini R, Serra G, Tognoni F. Colour changes of fresh-cut leafy vegetables during storage. Food Agriculture and Environment 2004;2:4044.

9. Fu H, Xie B, Ma S, Zhu X, Fan G, Pan S. Evaluation of antioxidant activities of principal carotenoids vailable in water spinach (Ipomoea aquatica). J Food Compos. Anal 2011;24:288-297.

10. Garavand F, Rahaee S, Vahedikia N, Jafari SM. Different techniques for extraction and micro/nanoencapsulation of saffron bioactive ingredients. Trends in Food Science and Technology 2019;89:26-44. Scopus.

11. Goupy P, Hugues M, Boivin P, Amiot MJ. Antioxidant composition and activity of barley (Hordeum vulgare) and malt extracts and of isolated phenolic compounds. J Sci. Food Agric 1999;79:1625-1634.

12. Harwansh RK, Deshmukh R, Rahman MA. Nanoemulsion: Promising nanocarrier system for delivery of herbal bioactives. Journal of Drug Delivery 
Science and Technology 2019;51:224-233. Scopus. https://doi.org/10.1016/j.jddst.2019.03.006.

13. INRA. INRA (Institut National de la Recherche Agronomique) Phenol Explorer Database 2009. Available at: http://www.phenol explorer.eu/.

14. Kolayli S, Kara M, Tezcan F, Erim FB, Sahin H, Ulusoy E, Aliyazicioglu R. Comparative study of chemical and biochemical properties of different melon cultivars: standard, hybrid, and grafted melons. Journal of Agricultural and Food Chemistry 2010;58:9764-9769.

15. Oliveira VS, Augusta IM, Braz MV, Riger CJ, Prudêncio ER, Sawaya ACHF et al. Aroeira fruit (Schinus terebinthifolius Raddi) as a natural antioxidant: Chemical constituents, bioactive compounds and in vitro and in vivo antioxidant capacity. Food Chemistry 2020, 126274.

16. Laur LM, Tian L. Provitamin A and vitamin C contents in selected California-grown cantaloupe and honeydew melons and imported melons. J Food Comp. Anal 2011;24:194-201.

17. Mattila P, Astola J, Kumpulainen J. Determination of flavonoids in plantmaterial by HPLC with diode-array and electro-array detection. J Agric. Food Chem 2000;48:5834-584.

18. Mesquita LM, Martins M, Maricato É, Nunes C, Quinteiro PS, Dias AC et al. Ionic liquid-mediated recovery of carotenoids from the Bactris gasipaes fruit waste and their application in food-packaging chitosan films. ACS Sustainable Chemistry and Engineering 2020.

19. Min S, Jin T, Yeom HW, Min SK, Zhang QH. Commercialscale pulsed electric field processing of orange juice. J Food Sci. Technol 2003;68:1265-1271. https://doi.org/10.1111/j.1365-2621.2003.tb09637.x

20. Meydov S, Saguy I, Kopelman IJ. Browning determination in citrus products. J Agric. Food Chemistry 1977;25(3):602.

21. Mokhtar SM, Ibrahim IMA. Physicochemical, Antioxidant and Sensorial Properties of Pasteurized Guava Nectar Incorporated with Pomegranate Peel and Guava Leaf Extracts. World Journal of Food Science and Technology 2020;4(1):8-16.

22. Murador DC, Mesquita LM, Vannuchi N, Braga ARC, Rosso VVD. Bioavailability and Biological Effects of Bioactive Compounds Extracted with Natural Deep Eutectic Solvents and Ionic Liquids: Advantages Over Conventional Organic Solvents. Current Opinion in Food Science 2019.https://doi.org/10.1016/j.cofs.2019.03.00 2.

23. Paul S, Geng C, Yang T, Yang Y, Chen J. Phytochemical and Health Beneficial Progress of Turnip (Brassica rapa). Journal of Food Science 2019;84(1):19-30.

24. Rabie MA, Soliman AZ, Diaconeasa ZS, Constantin B. Effect of pasteurization and shelf life on the physicochemical properties of physalis (Physalis peruviana L.) juice. Journal of Food Processing and Preservation 2015;39:1051-1060.

25. Ravichandran K, Saw NMMT, Mohdaly AA, Gabr AM, Kastell A, Riedel $\mathrm{H}$ et al. Impact of processing of red beet on betalain content and antioxidant activity. Food Res. Int 2013;50:670-675. https://doi.org/10.1016/j. foodres.2011.07.002

26. Santamarina AB, Jamar G, Mennitti LV, Ribeiro DA, Cardoso CM, De Rosso VV et al. Polyphenols-Rich Fruit (Euterpe edulis Mart.) Prevents Peripheral Inflammatory Pathway Activation by the Short-Term High-Fat Diet. Molecules 2019;24:1655. https://doi.org/10.3390/ molecules 24091655 .
27. Sharma P, Choudhary R, Thakur NS, Thakur A. Development and quality of apple -whey based herbal functional ready-to-serve beverage. Journal of Applied and Natural Science 2019;11(2):291-298.

28. Spadafora ND, Amaro AL, Pereira MJ, Müller CT, Pintado M, Rogers HJHJ. Multi trait approach to evaluate physiological and chemical interactions underlying flavour perception of stored rocket leaves. Food Chemistry 2016;211:114-123.

29. Sroy S, Fundo JF, Miller FA, Brandão TRS, Silva CLM. Impact of ozone processing on microbiological, physicochemical, and bioactive characteristics of refrigerated stored Cantaloupe melon juice. J Food Process Preservation 2019;43:1-9.

30. Tahar HS, Hamid G, Rachid HB, Souad H. Evolution of Bioactive Components of Prickly Pear Juice (Opuntia ficus indica) and Cocktails with Orange juice. Nature and Technology Journal, Vol. B, Agronomic and Biological Sciences 2019;20:45-53. http://www.univchlef.dz/revuenatec/issue-20/Article_B/Article_499.pdf

31. Tomas-Barberan FA, Clifford MN. Dietary hydroxybenzoic acid derivatives and their possible role in health protection. Journal of the Science of Food and Agriculture 2000;80:1024-1032.

32. Wang R, Tian Z, Chen L. Nano-encapsulations liberated from barley protein micro particles for oral delivery of bioactive compounds. International Journal of Pharmaceutics 2011;406(1-2):153-162. Scopus. https:// doi.org/10.1016/j.ijpharm.2010.12.039

33. Wibowo S, Vervoort L, Tomic J, Santiago JS, Lemmens L, Panozzo A et al. Colour and carotenoid changes of pasteurised orange juice during storage. Food Chemistry 2015;171:330-340. 